\title{
Photon and polariton fluctuations in arrays of QED-cavities
}

\author{
-Davide Rossini ${ }^{1}$, Rosario Fazio ${ }^{1,2}$ and Giuseppe Santoro ${ }^{1,3,4}$
}

International School for Advanced Studies (SISSA), Via Beirut 2-4, I-34014 Trieste, Italy

NEST-CNR-INFM \& Scuola Normale Superiore, Piazza dei Cavalieri 7, I-56126 Pisa, Italy

CNR-INFM Democritos National Simulation Center, Via Beirut 2-4, I-34014 Trieste, Italy

4 International Centre for Theoretical Physics (ICTP), P.O.Box 586, I-34014 Trieste, Italy

PACS 71.36.+c - Polaritons

PACS 73.43.Nq - Quantum phase transitions

PACS 42.50.Ct - Quantum description of interaction of light and matter

\begin{abstract}
We propose to detect the Mott insulator-superfluid quantum phase transition in an array of coupled cavities by studying the polariton and photon fluctuations in a block of linear dimension $M$ (in units of the lattice constant of the array). We explicitly show this for a one-dimensional array; the analysis can be however extended to higher dimensions. In the Mott phase polariton fluctuations are independent of the block size. In the superfluid phase they grow logarithmically with $M$, the prefactor being related to the compressibility of the system. In the case of photon fluctuations, the critical behaviour is encoded in the subleading scaling with the block dimension, while the leading behaviour is linear in $M$ and non-critical. Our results have been obtained by means of the density matrix renormalization group numerical algorithm.
\end{abstract}

The recent suggestion [1-3] to realize Mott and superfluid phases in arrays of coupled QED-cavities has stimulated a flurry of activity on strongly interacting photonic systems [4-12]. In the presence of randomness a glassy phase of polaritons was shown to appear in the phase dia-gram [5]. Coupled cavities can be engineered to behave as quantum simulators for a variety of interacting spin models $[7,9,10]$; they can support soliton excitations $[6,13]$. A different realization of the strongly interacting regime with photons in an optical guide was proposed in [14], while polariton blockade effects were studied in a resonantly excited photonic quantum dot [15]. As candidates for simulating strongly interacting models, coupled cavities present new characteristics as compared to other systems, like optical lattices [16] or Josephson junction arrays [17]. Most notably, it is possible to access their local properties.

In this paper we would like to exploit the local addressability of cavity arrays to propose a method in order to probe the critical behaviour of the Mott insulatorsuperfluid transition. Our suggestion is based on the study of the fluctuations in the number of photons and polaritons. To our knowledge, this issue was not addressed so far in the literature. While it is well known how to distinguish a regime of photon blockade, how to detect the transition itself is, to a large extent, an unexplored problem. We study in details a one-dimensional array. Our conclusions, however, can be easily extended to higher dimensions.

The Model - We suppose that inside each cavity a single two-level atom interacts with photons via a JaynesCummings Hamiltonian [18]. While this situation is simpler to simulate, we do not expect, for the purposes of our work, any qualitative change if other atomic level structures [1] are considered. The Hamiltonian for an array of $L$ coupled cavities is then given by

$\mathcal{H}=\sum_{i=1}^{L}\left[\epsilon \sigma_{i}^{+} \sigma_{i}^{-}+\omega a_{i}^{\dagger} a_{i}+\beta\left(\sigma_{i}^{+} a_{i}+\right.\right.$ h.c. $\left.)\right]-t \sum_{\langle i, j\rangle} a_{i}^{\dagger} a_{j}$

where $\epsilon$ denotes the transition energy between the two atomic levels, $\omega$ is the resonance frequency of the cavity, $\beta$ is the atom-field coupling constant $(\epsilon, \omega, \beta>0)$, and $t$ is the inter-cavity photon hopping, that is assumed to be constant for all nearest neighbours and zero otherwise. The atomic and photonic raising/lowering operators are denoted as $\sigma_{i}^{ \pm}$, and $\left\{a_{i}^{\dagger}, a_{i}\right\}$ respectively, the subscript $i$ indicating the lattice site. The total number of atomic plus photonic excitations (i.e., the number operator for polaritons) on the $i$-th site is given by $n_{i}^{\mathrm{pol}}=n_{i}^{\mathrm{ph}}+\sigma_{i}^{+} \sigma_{i}^{-}$, where $n_{i}^{\mathrm{ph}}=a_{i}^{\dagger} a_{i}$ is the photon number operator. Our analysis is restricted to the case of zero relative detuning 
$\Delta \equiv \omega-\epsilon$; we also work in the canonical ensemble with a fixed polariton density $\rho \equiv N / L=1, N$ being the total number of polaritons in $L$ cavities.

The equilibrium phase diagram associated to the model in Eq. (11) is characterized by two distinct phases, with polariton Mott Insulating (MI) regions surrounded by the Superfluid (SF) phase. In the MI phase polaritons are localized on each site due to the photon blockade [19], and there is a gap in the spectrum. A finite hopping renormalizes this gap, which eventually vanishes at a critical value of $t$. For large hoppings excitations are delocalized and the system enters the SF phase.

We propose to detect the MI-SF transition by analyzing the fluctuations of the occupation number inside a block composed by a subset of $M$ adjacent cavities. Since the number operator is the canonically conjugated variable with respect to the phase of the whole many-body wavefunction, we expect that its fluctuations are strongly suppressed in the incoherent MI regime, and, by contrast, greatly enhanced in the coherent SF phase. The dispersion of particle number on a given subsystem with $M$ sites is quantified by the variance of the corresponding probability distribution:

$$
\delta n_{\alpha}^{2}(M)=\left\langle\left(\sum_{i \in M} n_{i}^{\alpha}\right)^{2}\right\rangle-\left\langle\sum_{i \in M} n_{i}^{\alpha}\right\rangle^{2}
$$

where $\alpha=\mathrm{pol} / \mathrm{ph}$ stands for polariton/photon fluctuations, and $\langle\cdot\rangle$ denotes an average on the system ground state. This can be obtained from the two-point correlation functions of the related number operator: $C_{i j}^{\alpha} \equiv$ $\left\langle n_{i}^{\alpha} n_{j}^{\alpha}\right\rangle-\left\langle n_{i}^{\alpha}\right\rangle\left\langle n_{j}^{\alpha}\right\rangle$, such that $\delta n_{\alpha}^{2}(M)=\sum_{(i, j) \in M} C_{i j}^{\alpha}$.

In the present work we suppose that the system is in its ground state; we neglect decoherence, and assume that spontaneous photon emission and cavity loss characteristic times are much longer than the timescale over which the array can reach the ground state. We are aware that this is a strong assumption that may not be fulfilled in the experiments. We do not try to argue on this very important issue in the present work; rather we are interested in describing a method that is suitable to detect the various (equilibrium and non-equilibrium) many-body phases of an array of cavities. We describe our proposal by using the (equilibrium) MI-SF transition; the essential features of the method can be as well applied to the nonequilibrium case. We will come back to this point in the concluding section.

All the numerical data presented below have been obtained by means of the Density Matrix Renormalization Group (DMRG) algorithm with open boundary conditions.

Polariton fluctuations - We first study fluctuations in the polariton number. The number of polaritons inside a given subsystem can be experimentally measured by instantaneously switching off the effective polaritonic hopping (this can be achieved by changing the detuning $\Delta$, such to increase the relative strength of the atom-

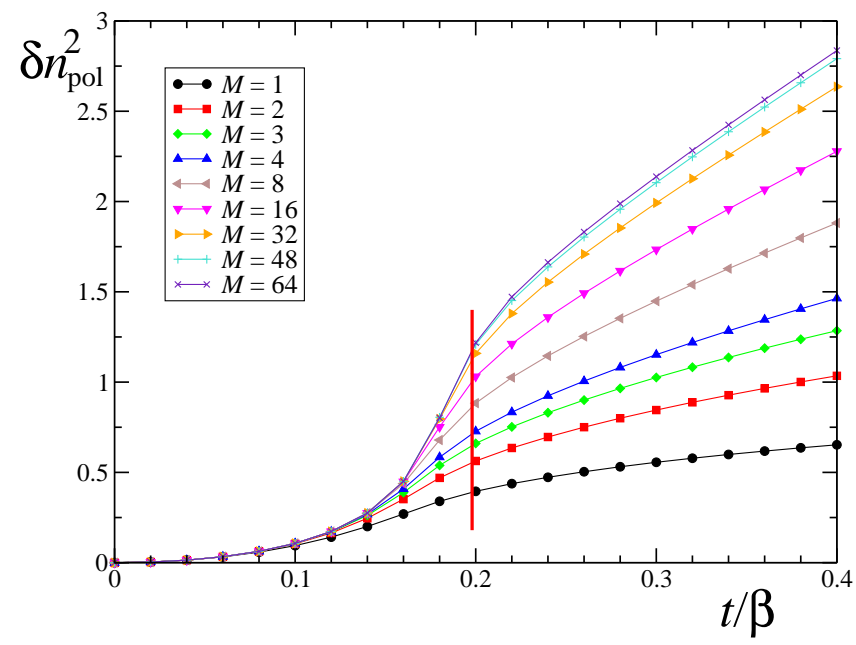

Fig. 1: (Colour on-line) Variance $\delta n_{\text {pol }}^{2}(M)$ of the probability distribution for the polaritonic occupation number inside a block of $M$ cavities as a function of the inter-cavity photon hopping $t$, for fixed values of $M$. With our choice of parameters, the MI-SF transition point is at $t^{*} / \beta \approx 0.198$ (red solid line). DMRG simulations have been performed for a system of $L=128$ cavities, keeping a maximum of $n_{\max }=10$ polaritons per site and a number $m=150$ of states; blocks are formed starting from the center of the chain.

field coupling with respect to the photon hopping). In this way, admitting radiative losses on long time scales, a quantum-jump picture immediately suggests that the polariton number is exactly given by the number of photons emitted from the selected region [20].

In Fig. 1] we show numerical data displaying $\delta n_{\text {pol }}^{2}(M)$ as a function of the photon hopping $t$ in a system with $L=128$ cavities; various curves correspond to different sizes $M$ of the block inside the system. The MI-SF transition point has been located numerically with high accuracy at $t^{*} / \beta \approx 0.198[5]$. On-site fluctuations cannot reveal critical features at the phase transition, because they correspond to a local property; whenever $t \neq 0$, the solid curve with circles corresponding to $M=1$ has always nonzero values. Therefore, an unambiguous characterization of the phase boundary is impossible in this context. This was already pointed out in Ref. [21] for the on-site boson number fluctuations in the Bose-Hubbard model, where it has been shown that the number probability distribution evolves from a Poissonian, in the noninteracting gas, to a sharply peaked distribution, in the insulator. We obtained very similar distribution probabilities for the on-site polariton number in our system, that are characterized by sub-Poissonian statistics.

From Fig. 1 we notice that, by increasing $M$, in the MI phase fluctuations tend to be independent on the block size, while in the SF phase the dependence on $M$ is evident. In particular, we found that $\delta n_{\text {pol }}^{2}(M)$ as a function of $M$ saturates to a finite constant value in the MI phase, while it diverges logarithmically in the SF phase (see in- 
set of Fig. 2, where we plotted the same curves of Fig. 1. but fixing $t$ and varying $M$ ). The logarithm divergence is directly related to the fact that density correlation functions in the superfluid phase have a power-law decaying uniform term [22]:

$$
C_{r}^{\mathrm{pol}}=\left\langle n_{i+r}^{\mathrm{pol}} n_{i}^{\mathrm{pol}}\right\rangle-\left\langle n_{i+r}^{\mathrm{pol}}\right\rangle\left\langle n_{i}^{\mathrm{pol}}\right\rangle \sim \frac{2}{K}(2 \pi \rho r)^{-2}+\cdots
$$

In the previous expression $\rho$ is the particle (in this case the polariton) density and $K$ is the so called Luttinger parameter [23], that is proportional to the square root of the compressibility of the system. Indeed, from the definition of $\delta n_{\text {pol }}^{2}(M)$, one immediately concludes that, in the SF phase

$$
\delta n_{\text {pol }}^{2}(M) \sim \frac{1}{K \pi^{2} \rho^{2}} \ln M .
$$

At the SF-MI transition the coefficient $K$ at integer densities is known to be equal to $K_{c}=1 / 2$ [23]. Therefore, for a polariton density $\rho=1$, the logarithmic prefactor suddenly jumps from a value $\bar{c}_{0}=2 / \pi^{2}$ just inside the $\mathrm{SF}$ phase, to zero in the MI, with a characteristic KosterlitzThouless behaviour.

A quantitative analysis of the crossover between the two different behaviours has been performed by fitting numerical data according to:

$$
\delta n_{\mathrm{pol}}^{2}(M)=c_{0}^{\mathrm{pol}} \ln \left[\frac{L}{\pi} \sin \left(\frac{\pi}{L} M\right)\right]+A^{\mathrm{pol}},
$$

with $A^{\text {pol }}$ and $c_{0}^{\text {pol }}$ as fitting parameters. The constant term $A^{\text {pol }}$ is not important for our purposes, therefore we concentrate on the logarithmic term. In the main panel of Fig. 2 we plot the logarithmic prefactor $c_{0}^{\text {pol }}$ as a function of $t$ : the phase transition point in this situation can be quite clearly identified. In $1 \mathrm{D}$, in particular, a precise criterion for values of $c_{0}^{\text {pol }} \geq 2 / \pi^{2}$ in the superfluid phase, clearly identifies the transition point.

Photon fluctuations - We now concentrate on the photon number fluctuations, a quantity which is more directly measured in quantum optical experiments, where the system is typically in a non-equilibrium state between photon/cavity decay and external pumping. The state of the polariton field is usually retrieved by detecting and characterizing the light emission [24,25].

In this case the situation is quite different as compared to the case of polariton fluctuations. Even at zero hopping the on-site photon number is fluctuating. For example, in the case of zero relative detuning $(\Delta=0)$ and deep in the MI regime, we have $\left\langle n_{i}^{\mathrm{ph}}\right\rangle \approx 1 / 2$ and $\left\langle\left(n_{i}^{\mathrm{ph}}\right)^{2}\right\rangle \approx 1 / 2$. Therefore, even for a perfect insulator, the variance $\delta n_{\mathrm{ph}}^{2}(M)$ of the photon number distribution inside a block of length $M$ is proportional to the block size: $\delta n_{\mathrm{ph}}^{2}(M) \approx M / 4$. The onset of the superfluid behaviour can then be sought in the deviations from this linear growth. These deviations are due to the raising of

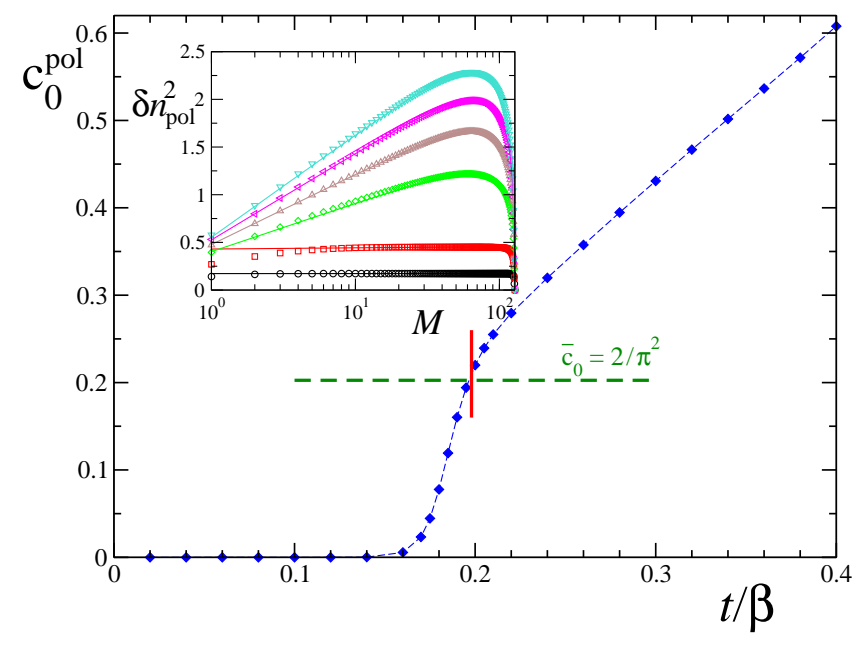

Fig. 2: (Colour on-line) Best fitting parameter $c_{0}^{\text {pol }}$ of Eq. (5) for the variance $\delta n_{\text {pol }}^{2}(M)$ as a function of the hopping $t$. The horizontal dashed line indicates the estimate of the logarithmic prefactor at the transition point: $\bar{c}_{0} \sim 2 / \pi^{2}$. In the inset we plot $\delta n_{\mathrm{pol}}^{2}$ as a function of $M$ (symbols) for some fixed values of $t$ : from bottom to top $t=0.12,0.16,0.2,0.24,0.28,0.32$; continuous lines are logarithmic fits of the corresponding data. In numerical fits we dropped the first and the latter 10 points (i.e., we kept values for $M \in[11, L / 2-10]$ ).

correlations between distant sites on increasing the hopping strength. Therefore, in the scaling ansatz for the photon fluctuations one should also include a term that is linear in the block dimension, i.e.

$$
\delta n_{\mathrm{ph}}^{2}(M)=\alpha M+c_{0}^{\mathrm{ph}} \ln \left[\frac{L}{\pi} \sin \left(\frac{\pi}{L} M\right)\right]+A^{\mathrm{ph}} .
$$

Let us analyze the properties of the first two terms in the r.h.s. of Eq. (6). The behaviour of $\alpha$ as a function of the hopping is shown in Fig 3. Starting from the value $\alpha=1 / 4$ at $t=0$, the coefficient of the linear term decreases on increasing the hopping. This behaviour signals the fact that correlations between photon fluctuations at different locations start to develop. Although at $t \sim 0.2$ there is an indication of the change in the ground state properties, the coefficient $\alpha$ seems not to be an appropriate indicator of the location of the critical point. As for the case of polariton fluctuations, also in the case of photons one should look at the coefficient of the logarithmic term. This is shown in Fig. 4 here the critical behaviour is easily identified. The value of the fluctuation at the transition is not universal as in the polariton case; the reason is that photons are not the genuine excitations of the system, therefore the considerations leading to Eq. (4) with $K_{c}=1 / 2$ do not apply. The ratio between the coefficients of polariton and photon fluctuations is shown in the inset of Fig. 4. A marked change at the transition can be noticed. In our opinion, however, this is not obviously related to the critical point; rather we expect that the ratio is a non-universal feature depending on the details of the model and on the value of the couplings. 


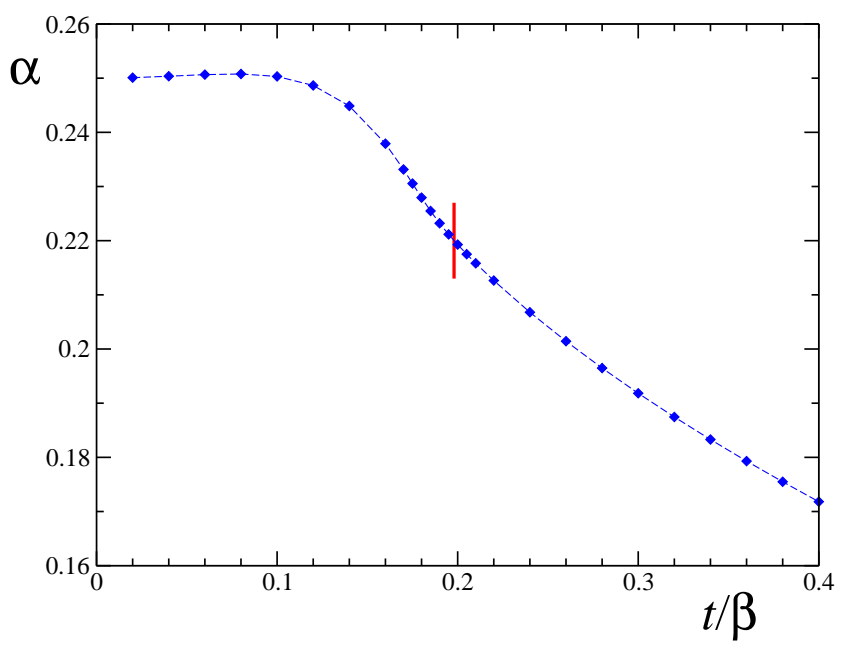

Fig. 3: (Colour on-line) Coefficient of the linear term in the photon fluctuations, see Eq. (6), as a function of the photon hopping $t$ between the cavities. As it is evident from the plot, $\alpha$ carries no information about the critical behaviour (as a reference, we indicated with a red bar the location of the critical point).

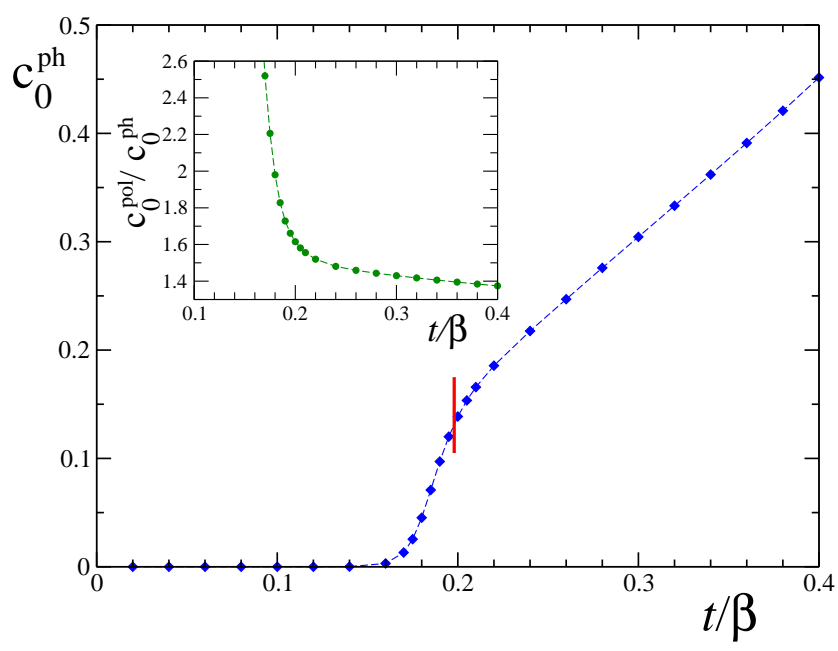

Fig. 4: (Colour on-line) Prefactor $c_{0}^{\mathrm{ph}}$ of the logarithmic term in Eq. (6) as a function of the hopping. The behaviour is very similar to the corresponding prefactor for the polariton fluctuations. The ratio between $c_{0}^{\mathrm{pol}}$ and $c_{0}^{\mathrm{ph}}$, shown in the inset, is non-universal.

Indication of the critical behaviour from photon correlations may also be obtained without resorting to a scaling analysis as a function of the block size $M$. This can be achieved by studying the second derivative of photon number fluctuations with respect to the block size, evaluated for blocks of half the length of the system size, that is $\left.\partial_{M}^{2}\left[\delta n_{\mathrm{ph}}^{2}(M)\right]\right|_{M=L / 2}$. Although this approach might be slightly less accurate, it can give an interesting insight into the critical region.

We first consider $\left.\partial_{M}^{2}\left[\delta n_{\mathrm{ph}}^{2}(M)\right]\right|_{M=L / 2}$ as a function of the hopping parameter $t$. Numerical data in Fig. 5 show

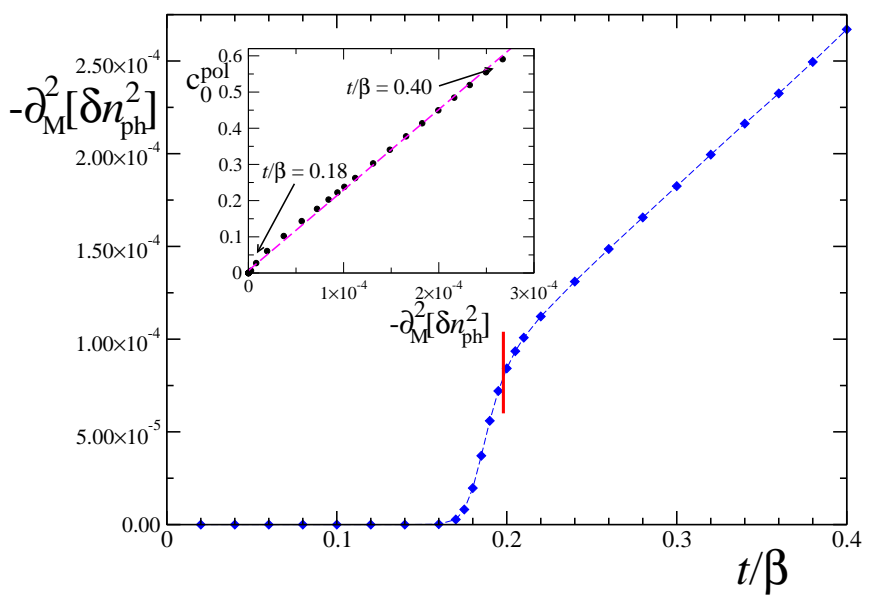

Fig. 5: (Colour on-line) Second derivative of photon number fluctuations with respect to the block length, evaluated at half the length of the system size, as a function of the hopping $t$. Inset: logarithmic prefactor of polariton number fluctuations, as a function of the second derivative of photon number fluctuations (data are the same of the main panel and of Fig. 2).

that, like for the polaritonic number fluctuations, also this quantity can be used to characterize the MI-SF transition for finite sizes. In addition, we can identify a behaviour that is very similar to the one of the logarithmic prefactor $c_{0}^{\mathrm{pol}}$ of Eq. (5) for the polariton number fluctuations. Namely, the correlation between these two quantities is nearly perfect: apart from a proportionality factor, which depends on the system size, the two curves in the main panels of Figs. 2 2 are exactly the same. This is shown in the inset of Fig. 5 where, for a given value of the photon hopping $t$, we plot the corresponding values of $c_{0}^{\mathrm{pol}}$ and of $\left.\partial_{M}^{2}\left[\delta n_{\mathrm{ph}}^{2}(M)\right]\right|_{M=L / 2}$ in the two axes, thus displaying a perfect linear correlation.

The dependence of the photon fluctuations on the size is however more complicated than that of $\delta n_{\mathrm{pol}}^{2}$. In Fig. 6 we plot $\left.\partial_{M}^{2}\left[\delta n_{\mathrm{ph}}^{2}(M)\right]\right|_{M=L / 2}$ (its absolute value, actually, since it is always negative) as a function of the system size $L$, for several fixed photon hoppings $t$. While the logarithmic prefactors $c_{0}^{\mathrm{pol}}$ and $c_{0}^{\mathrm{ph}}$ are independent of the system size, this is not the case for the second derivative of photon number fluctuations, which asymptotically drops to zero as a power-law with $L$. More specifically, in the free photon limit $(t \rightarrow+\infty)$ it decays as $L^{-1}$, while for finite values of the photon hopping and a sufficiently large system size, there is a crossover to a $L^{-2}$ behaviour. This follows from two qualitatively different decays of the photon number correlation function $C_{r}^{\mathrm{ph}}=\left\langle n_{i+r}^{\mathrm{ph}} n_{i}^{\mathrm{ph}}\right\rangle-\left\langle n_{i+r}^{\mathrm{ph}}\right\rangle\left\langle n_{i}^{\mathrm{ph}}\right\rangle$ : apart from open-boundary effects, in the first regime $C_{r}^{\mathrm{ph}} \approx 1 / L$, while in the second case $C_{r}^{\mathrm{ph}} \approx 1 / r^{2}$. We carried out a numerical analysis of the photon correlations $C_{r}^{\mathrm{ph}}$ in our QED-cavity array system, and explicitly found these two distinct behaviours.

It has also been possible to derive an analytic estimate of the crossover scale by exploiting a mapping of the cavity 


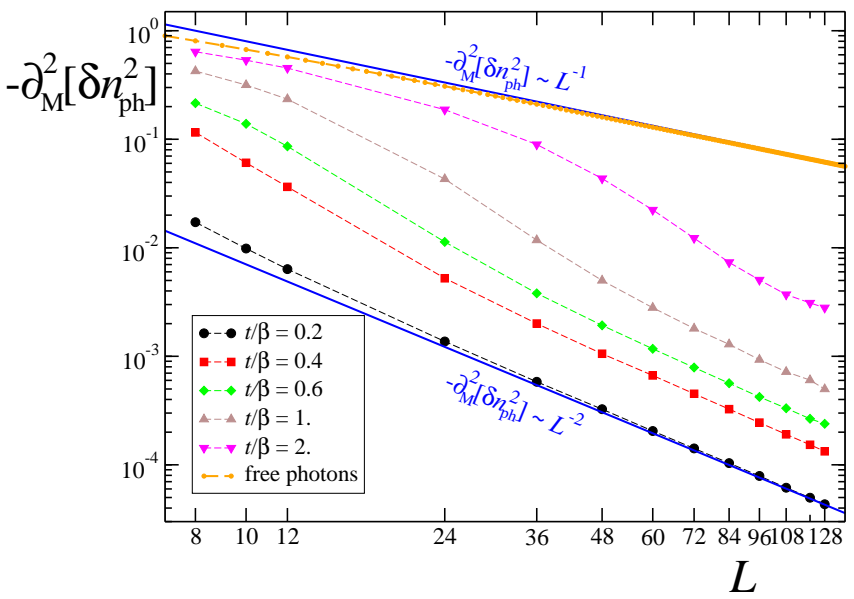

Fig. 6: (Colour on-line) Second derivative of photon number fluctuations with respect to the block size $M$ evaluated at $M=L / 2$, as a function of the system size $L$. The two straight blue lines denote power-law behaviours $L^{-1}, L^{-2}$. DMRG parameters are the same as in Figs. 1 and 2

array with a Bose-Hubbard (BH) model, and studying its corresponding boson correlators $C_{i j}^{\mathrm{BH}}$. It should be kept in mind that the formal analogy between $C_{i j}^{\mathrm{ph}}$ and $C_{i j}^{\mathrm{BH}}$ has to be considered only at a qualitative level, since the equivalence of the two models becomes exact only in the limit of a large number of atoms per cavity [1].

The BH Hamiltonian is defined by

$$
\mathcal{H}=-J \sum_{j}\left(b_{j+1}^{\dagger} b_{j}+\text { h.c. }\right)+\frac{U}{2} \sum_{j=1}^{L} b_{j}^{\dagger} b_{j}^{\dagger} b_{j} b_{j},
$$

where $\left\{b_{j}^{\dagger}, b_{j}\right\}$ are the boson creation/annihilation operators, $n_{i}=b_{i}^{\dagger} b_{i}$ the boson number operator, and with $C_{i j}^{\mathrm{BH}}$ we indicate the corresponding correlation functions. When the depletion of the condensate is not too great $(J \gg U)$, we can employ the Bogoliubov approximation, which consists in replacing the boson creation and annihilation operators at a given site $j$ by a c-number $z_{i} \in \mathbb{C}$ plus a fluctuation operator $\beta_{j}$. Only terms at most of the second order in $\beta_{j}$ are considered when diagonalizing the Hamiltonian [26-28]. Within this framework and for periodic boundary conditions, we locate the crossover scale between the $L^{-1}$ and $L^{-2}$ behaviour by

$$
U / J \lesssim \frac{2 \pi^{2}}{n_{0} L^{2}}
$$

with $n_{0}$ being the density of the boson condensate. Whenever the inequality is valid, the spectrum of the Bogoliubov quasi-particles at small $k$ is quadratic, and correlations behave as for free bosons: $C_{r}^{\mathrm{BH}} \approx 1 / L$. Conversely, if it does not hold, the spectrum becomes linear at small $k$, and the free-boson limit fails; in this regime we recover the decay $C_{r}^{\mathrm{BH}} \approx 1 / r^{2}$ for large $r$, typical of the polariton number correlations $[22]$.
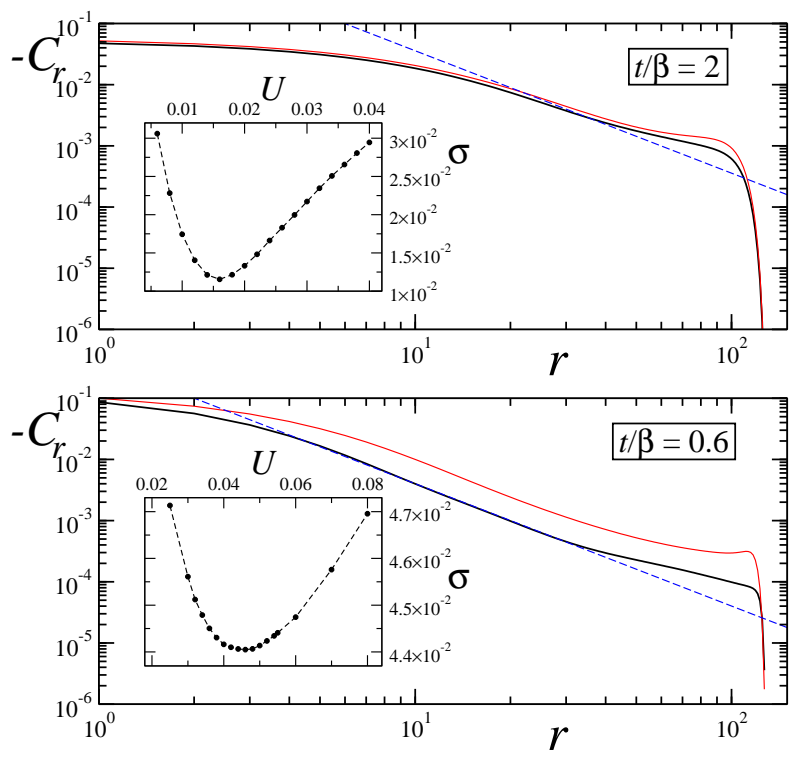

Fig. 7: (Colour on-line) Number fluctuations of photons $C_{r}^{\mathrm{ph}}$ in an array of cavities (black curves - DMRG data) and of bosons $C_{r}^{\mathrm{BH}}$ in BH model (red curves - Bogoliubov ansatz); dashed blue curves display a behaviour $C_{r} \sim r^{-2}$. The two panels correspond to different values of the hopping, that is assumed to be equal in the two models $t=J$. In the insets we plot the deviation $\sigma$ of correlations for the two models, as a function of $U$; the red curve in each panel shows the best fitting curve of DMRG data, corresponding to the value of $U$ which minimizes $\sigma$. We analyzed chains of $L=128$ sites, and evaluated correlations starting from the central site.

A proper quantitative comparison between photon number correlations in the coupled cavity system and boson number correlations in the $\mathrm{BH}$ has been obtained by numerically solving the $\mathrm{BH}$ Bogoliubov equations for a system with open boundaries, and it is shown in Fig. 7 The hopping is chosen to be equal in the two models $(t=J)$; the polariton/boson density is $\rho=1$. DMRG data are fitted by varying the on-site repulsion $U$ in the $\mathrm{BH}$ and by minimizing the squared differences between the two curves: $\sigma^{2}(U)=\sum_{r}\left|C_{r}^{\mathrm{ph}}-C_{r}^{\mathrm{BH}}\right|^{2}$. Of course, if $t / \beta$ is too small the Bogoliubov approximation fails, and we do not find an appropriate minimum. We remark that correlations are always negative, thus indicating photon antibunching, as it is revealed by on-site number distributions with negative values of the Mandel parameter [18].

Conclusions - In this paper we studied photon and polariton fluctuations in coupled cavities. By a suitable scaling of the fluctuation detected over a region of the sample, we showed that it is possible to extract the critical properties of the superfluid to Mott insulator phase transition. The analysis presented here for one-dimensional systems can be extended to higher dimensional lattices. We remark that we confined our study to the equilibrium case; an interesting point that remains to be addressed is to consider the effects of photon and cavity losses, which may lead to new non-equilibrium effects in the physics of such sys- 
tems. We do however expect that the method proposed here will hold also in the non-equilibrium case. The reason is that our approach ultimately distinguishes the system being incompressible or not. While the general principle also holds for driven systems, the detailed behaviour leading to the logarithmic scaling may be modified. As a consequence, the most appropriate scaling ansatz for the non-equilibrium cases has to be checked.

$$
* * *
$$

This work was supported by EU (EUROSQIP). We used the DMRG code of the "Powder with Power" project (http://www.qti.sns.it/). We thank M. B. Plenio and I. Carusotto for interesting discussions.

\section{REFERENCES}

[1] Hartmann M. J., Brandão F. G. S. L. and Plenio M. B., Nature Phys., 2 (2006) 849.

[2] Greentree A. D., Tahan C., Cole J. H. and HollenBerg L. C. L., Nature Phys., 2 (2006) 856.

[3] Angelakis D. G., Santos M. F. and Bose S., Phys. Rev. A, 76 (2007) 031805(R).

[4] Neil Na Y.C., Utsunomiya S., Tian L. and Yaмамото Y., Phys. Rev. A, 77 (2008) 031803(R).

[5] Rossini D. and Fazio R., Phys. Rev. Lett., 99 (2007) 186401.

[6] Paternostro M., Agarwal G. S. and Kim M .S., arXiv:0707.0846 preprint, 2007.

[7] Hartmann M. J., Brandão F. G. S. L. and Plenio M. B., Phys. Rev. Lett., 99 (2007) 160501.

[8] Hartmann M. J. and Plenio M. B., Phys. Rev. Lett., 99 (2007) 103601.

[9] Cho J., Angelakis D. G. and Bose S., arXiv:0802.3365 preprint, 2008.

[10] KAY A. and Angelakis D. G., arXiv:0802.0488 preprint, 2008.

[11] Makin M. I., Cole J. H., Tahan C., Hollenberg L. C. L. and Greentree A. D., Phys. Rev. A, 77 (2008) 053819 .

[12] Aichhorn M., Hohenadler M., Tahan C. and LitTlewood P. B., Phys. Rev. Lett., 100 (2008) 216401.

[13] Slusher R. E. and Eggleton B. J., Nonlinear Photonic Crystals (Springer, Berlin) 2003.

[14] Chang D. E., Gritsev V, Morigi G., Vuletić V., Lukin M. D. and Demler E. A., arXiv:0712.1817 preprint, 2007.

[15] Verger A., Ciuti C. and Carusotto I., Phys. Rev. B, 73 (2006) 193306.

[16] Lewenstein M., Sanpera A., Ahufinger V., Damski B., Sen(De) A. and Sen U., Adv. Phys., 56 (2007) 243.

[17] Fazio R. and van der Zant H., Phys. Rep., 355 (2001) 235.

[18] Shore B. W. and Knight P. L., J. Mod. Opt., 40 (1993) 1195.

[19] Imamō̄lu A., Schmidt H., Woods G. and Deutsch M., Phys. Rev. Lett., 79 (1997) 1467; ReBić S., TAN S. M., Parkins A. S. and Walls D. F., J. Opt. B, 1 (1999) 490; Kim J., Benson O., Kan H. and Yamamoto Y.,
Nature, 397 (1999) 500; Birnbaum K. M., Boca A., Miller R., Boozer A. D., Northup T. E. and Kimble H. J., Nature, 436 (2005) 87.

[20] Plenio M. B. and Knight P. L., Rev. Mod. Phys., 70 (1998) 101.

[21] Capogrosso-Sansone B., Kozik E., Prokof'ev N. and Svistunov B., Phys. Rev. A, 75 (2007) 013619.

[22] Haldane F. D. M., Phys. Rev. Lett., 47 (1981) 1840.

[23] Giamarchi T., Phys. Rev. B, 46 (1992) 342.

[24] Carusotto I. and Ciuti C., Phys. Rev. B, 72 (2005) 125335.

[25] Verger A., Carusotto I. and Ciuti C., Phys. Rev. B, 76 (2007) 115324.

[26] van Oosten D., van der Straten P. and Stoof H. T. C., Phys. Rev. A, 63 (2001) 053601.

[27] Rey A. M., Burnett K, Roth R., Edwards M., Williams C. J. and Clark C. W., J. Phys. B: At. Mol. Opt. Phys., 36 (2003) 825.

[28] Burnett K., Edwards M., Clark C. W. and Shotter M., J. Phys. B: At. Mol. Opt. Phys., 35 (2002) 1671. 